Rapid Response to Delivering cost effective healthcare through reverse innovation

\title{
Reverse innovation, power imbalances, language and avoiding cultural appropriation
}

Lokugamage AU, Douglass C, Gishen F, Fyffe M

BMJ (Clinical research ed.) 367:17003 17 Dec 2019

We thank Skopec, Issa and Harris(1) for their fascinating essay on reverse innovation and note that it could herald an age of more cultural humility for the global north and assist with dissipating any prejudices towards the innovations from the global south. Bringing in new ideas from 'outside' requires disrupting entrenched power/ value systems around knowledge, which are inherent in the process of decolonising medicines and devices. Indeed, it chimes with Timms and Mearns book \#Newpower,(2) which refers to bottom up power surges (like \#MeToo) that are overturning old power hierarchies and protectionism around institutions.

However, we note that even though the authors did not coin the term reverse innovation, the terminology of 'reverse' conjures up notions of going backwards which could re-inforce power imbalances through language. Could there be a new term for this type of collaboration? Also, the pitfalls of cultural appropriation should be avoided where by aspects of an oppressed culture being taken out of context by a historically dominant people, who lack the cultural context to properly understand, respect, or utilise these elements.(3)

1. Skopec $M$, Issa $H$, Harris $M$. Delivering cost effective healthcare through reverse innovation. [cited 2019 Nov 28]; Available from: https://arbutusmedical.ca

2. Heimans J, Timms H. New power : how it's changing the 21st century - and why you need to know. 325 p.

3. Connecting up the curriculum - Decolonising the Medical Curriculum [Internet]. [cited 2019 Nov 28]. Available from:

https://decolonisingthemedicalcurriculum.wordpress.com/connecting-up-the-curriculum/ 